\title{
Descripción del accidente ofídico y manejo clínico en San Roque - Antioquia, por medio de revisión de historias clínicas, en el periodo de 2008 a 2014
}

\author{
Description of ophidian accidents and \\ clinical management in San Roque (Antioquia), \\ through the review of medical records for \\ the 2008-2014 period
}

1 Estudiantes décimo semestre de Medicina, Facultad de Ciencias de la Salud, Corporación Universitaria Remington, calle 51 \# 51-27, Medellín, Colombia. Correo: lauraremington15@gmail.com

2 Microbiólogo, MSc, integrante del Grupo de Investigaciones Biomédicas Uniremington, docente Facultad de Ciencias de la Salud, Corporación Universitaria Remington, calle 51 \# 51-27, Medellín, Colombia. Correo: edwin.higuita@uniremington.edu.co

3 Médica y cirujana, especialista en Toxicología Clínica, docente Facultad de Ciencias de la Salud, Corporación Universitaria Remington, calle 51 \# 51-27, Medellín, Colombia.
Laura Naranjo Bedoya`; Alejandra Parra Martínez ; Diber M. Romero Ayala

Tutores: Edwin A. Higuita David²; Isabel Escobar Toledo ${ }^{3}$

Resumen

En Colombia el accidente ofídico constituye un problema de salud pública que tiene su mayor incidencia en la zona rural. Presenta una prevalencia que va en aumento, indicando que ocurren tres mil accidentes por año entre envenenamientos y no envenenamientos; en gran parte de los pacientes la ausencia de información y reporte sobre el evento se debe a factores clínicos poco expresivos, ya que la mayoría de los casos son por envenenamientos leves, factores culturales que incluyen la medicina practicada por chamanes y curanderos, factores sanitarios como la falta de notificación y dificultades de acceso al sistema de salud como un factor geográfico de importancia. Además de esto, se ha observado un déficit de conocimientos en los médicos en cuanto al diagnóstico y tratamiento del ofidismo, así como en el adecuado manejo de los sueros antiofídicos. Se decide entonces caracterizar la clínica del accidente ofídico en el departamento de Antioquia y el manejo clínico que se le da en cada región mediante la revisión de historias clínicas en el periodo 2008-2014. Se encontró que la información dada a la comunidad, como promoción y prevención frente al accidente ofídico, sigue siendo deficiente. Se muestran estadísticas de las principales áreas corporales afectadas, asimismo, mediante la revisión y análisis de clasificación de los 
casos en relación con el tipo de manejo realizado, comparado con lo estipulado en manejo adecuado, se encuentra una falta de tratamiento idóneo y clasificación del caso por parte del personal; esto hace que sea necesario realizar autoevaluaciones y mejorar las capacitaciones del personal profesional.

Palabras clave: accidente ofídico, antiofídico, complicaciones, clasificación clínica, toxicidad.

\section{Abstract}

In Colombia ophidian accidents are an important public health problem that has its greatest impact on rural areas. An increasing prevalence is observed with 3,000 accidents occurring annually (with and without envenoming). For a significant proportion of patients, the lack of information and reporting on the event is due to poorly manifested clinical factors, since most cases correspond to mild envenomations; cultural factors, including treatments administered by shamans and healers; sanitary factors such as the lack of notification and geographical factors, such as difficulties in accessing the health system. In addition to this, insufficient knowledge among physicians has been observed in relation to diagnosis and treatment of ophidism, as well as to the proper management of antiophidian sera. Through the review of medical records for the 2008-2014 period, this study aimed to characterize the clinical assessment of ophidian-related accidents in the department of Antioquia as well as the clinical management provided in each region. The study found that the information provided to the community, including promotion and prevention of ophidian accidents, remains poor. Statistics about the main body areas affected are shown here. Also, upon examining and analysing the cases grading in relation to the type of management performed, compared to the provisions of appropriate management protocols, an inadequate treatment and envenomation grading of cases by the health care providers was found. These findings point to the need for carrying out self-assessments and enhacing the training of health care providers.

Keywords: ophidian accident, antiophidians, complications, clinical grading, toxicity.

\section{Introducción}

Los accidentes por mordeduras de animales ponzoñosos son una causa importante de envenenamiento a nivel mundial, ya que aproximadamente cinco millones de humanos pueden sufrirla anualmente, siendo mortales en $4.5 \%$ de los casos, y considerándose un problema de salud pública mundial (Campbell y Lamar, 2004). En las zonas tropicales de Latinoamérica anualmente ocurren 5,000 muertes asociadas a 150,000 envenenamientos, se considera que la población rural es la más afectada (Campbell y Lamar, 2004).

El accidente ofídico se define como la mordedura de serpientes que poseen e inoculan sustancias tóxicas, las cuales lesionan los tejidos y provocan alteraciones fisiopatológicas en la víctima (Campbell y Lamar, 2004). En Colombia, en la región Andina, principalmente Antioquia, y en el Caribe y la región Pacífica, principalmente el Chocó, son las zonas más fuertemente afectadas por dicha entidad clínica (Otero, 2007). La población más afectada son los agricultores hombres jóvenes con una tasa de $45.9 \%$ de los casos. Las extremidades inferiores son la zona del cuerpo más vulnerable a este tipo de ataques, y la especie Bothrops atrox es la principal serpiente asociada.

La mayoría de los venenos de serpientes (95\%) contienen una mezcla de proteínas y 
péptidos con aminoácidos, lípidos libres y carbohidratos; algunos venenos son proteolíticos y necrosantes (Bothrops y Lachesis) (De Lima et al., 2005; Fry y Wuster, 2004). Además, los principales tipos de accidentes ofídicos en Colombia son el bothrópico, lachésico y crotálico.

El diagnóstico de los accidentes ofídicos se realiza a través de: 1) La clínica, en la cual se busca la necrosis, edema, dolor, erita o la lesión visible dentro de la parte corporal donde se tuvo el contacto con el animal. 2) De acuerdo con la clasificación de los síntomas y el aspecto clínico que presenta el paciente al momento de diagnóstico, para conocer según las reglas promedio de veneno inoculado, el número de suero que requiere para inhibir la actividad tóxica de este (Cuesta, 2010).

Los pacientes pueden ser clasificados según sus estadios clínicos en: leve, moderado o severo, lo cual depende del tipo de accidente ofídico e, igualmente, según esta clasificación, se realiza el manejo con número de ampollas de suero antiofídico (Otero et al., 1992).

Son múltiples las complicaciones, las cuales pueden ser: necrosis y perdida del tejido, falla renal, síndrome compartimental, amputaciones, hemorragia, sepsis, alteraciones neurológicas y convulsiones, trombocitopenia y muerte (Cuesta et al., 2010; Otero et al., 1992; Otero et al., 2001; Otero et al., 2002).

Por lo anterior, se hace necesario caracterizar la clínica del accidente ofídico en el departamento de Antioquia y el manejo clínico que se le da en cada región, mediante la revisión de historias clínicas en el periodo 2008-2014, para realizar o estipular métodos que solucionen o mejoren el posible déficit encontrado en cada población.

\section{Materiales y métodos}

Tipo de estudio

Descriptivo retrospectivo de corte transversal.

\section{Población y muestra}

La población corresponde a los habitantes de San Roque, municipio de Colombia, ubicado en la región Nordeste del departamento de Antioquia, donde habitan 17,624 personas tanto de área urbana como de área rural; en el presente estudio se toman en cuenta habitantes que hayan estado expuestos a la mordedura de una serpiente.

La muestra corresponde a los pacientes atendidos en el hospital local del municipio por presencia de accidente ofídico por serpientes venenosas que cumplan con los criterios de inclusión y ninguno de exclusión. Se obtiene un número de 65 historias clínicas entre el año 2008 y 2014.

\section{Criterios de inclusión}

- Historias clínicas de pacientes residentes en el municipio de San Roque de manera permanente, que se desarrollen laboral y económicamente dentro del área.

- Historias clínicas de pacientes con clínica compatible con el accidente ofídico.

- Historias clínicas completas y adecuadas de pacientes que reporten el tiempo de hospitalización, secuelas inmediatas y posteriores, con encabezados apropiados que permitan la caracterización epidemiológica del paciente.

\section{Criterios de exclusión}

- Historias clínicas de pacientes remitidos a hospitales de mayor complejidad para el manejo del accidente ofídico en las primeras 6 horas de ocurrido el evento. 
- Historias clínicas de pacientes con secuelas no asociadas directamente al accidente ofídico.

- Historias clínicas de pacientes con inmunodeficiencias primarias o adquiridas identificadas previamente al evento.

Recolección de datos y análisis estadístico El plan de recolección de la información se realizó con la previa autorización de las directivas de la institución, basándonos en el Manual de ética médica, Código de bioética y bioseguridad del año 2002. Las variables de interés se consignaron en una base de datos diseñada para hacer los respectivos análisis que ayudaran a cumplir los objetivos propuestos. El análisis se realizó mediante el programa de Excel, utilizando valores porcentuales y desviación estándar.

\section{Resultados}

Se encontraron los siguientes resultados en el presente estudio, en el cual se recolectaron 65 historias correspondientes a pacientes, quienes sufrieron accidente ofídico en el municipio de San Roque - Antioquia y fueron atendidos en el hospital local en el periodo 2008-2014.

Más del $30 \%$ de los accidentes ofídicos se presentan en edad reproductiva teniendo como pico máximo los 30 años de edad. Estos accidentes afectan aún más al sexo masculino y se presentan con mayor frecuencia en el área rural; esta proporción corresponde a un $85 \%$ (ver Tabla 1 y Figura 1).
Tabla 1. Descripción sociodemográfica.

\begin{tabular}{|c|c|c|}
\hline Edad & Media & $\mathbf{3 5 . 4 9}$ \\
\hline & Desviación estándar & 18.75 \\
\hline Sexo & Hombre & $75.38 \%$ \\
\hline Ocupación & Mujer & $24.61 \%$ \\
\hline & Estudiante & $10.76 \%$ \\
\hline & Agricultor & $66.15 \%$ \\
\hline & Jornalero & $6.15 \%$ \\
\hline & Hogar & $7.69 \%$ \\
\hline & Otros & $6.15 \%$ \\
\hline
\end{tabular}

Fuente. Elaboración propia.

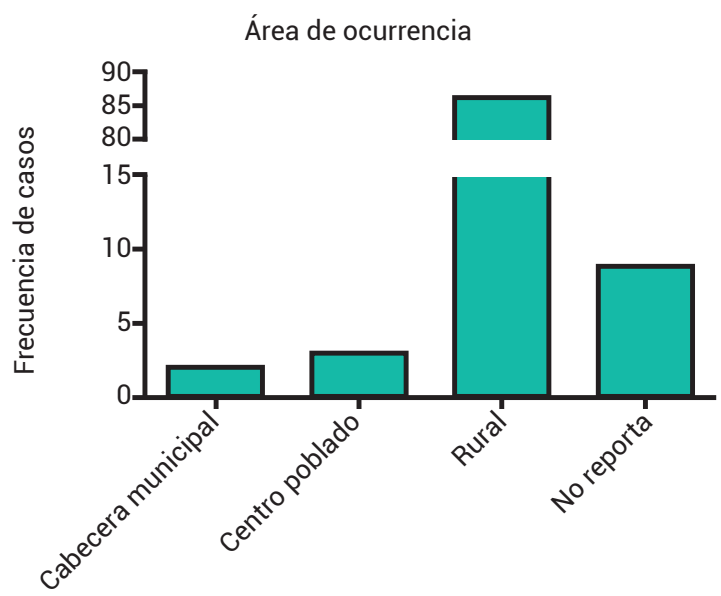

Figura 1. Área de ocurrencia del accidente ofídico en el municipio de San Roque.

Fuente. Elaboración propia.

Como tipo de atención inicial, la inmovilización del miembro es el único método preventivo para las posibles complicaciones que trae consigo el veneno del animal, según los últimos protocolos y circulares. En la muestra de población estudiada se evidencia que solo el $10.77 \%$ de los pacientes ingresados con accidente ofídico reciben este tipo de atención inicial (Tabla 2). 
Tabla 2. Tipo de atención inicial prehospitalaria.

\begin{tabular}{|l|c|}
\hline \multicolumn{1}{|c|}{ Tipo de atención pre hospitalaria } & Frecuencia (\%) \\
\hline Incisión & 7.69 \\
\hline Punción & 3.08 \\
\hline Inmovilización del miembro & 10.77 \\
\hline Succión bucal & 1.54 \\
\hline Sangría & 7.69 \\
\hline Succión mecánica & 0 \\
\hline Torniquetes & 29.23 \\
\hline Otros & 13.85 \\
\hline
\end{tabular}

Fuente. Elaboración propia.

El recurso médico predomina sobre las prácticas no médicas como alternativas para el accidente ofídico; el $43.08 \%$ de la población afectada acudió al recurso médico sin realizar otras prácticas (Tabla 3 ).

Tabla 3. Prácticas no médicas como atención inicial.

\begin{tabular}{|l|c|}
\hline \multicolumn{1}{|c|}{ Tipo de práctica no médica } & Frecuencia (\%) \\
\hline Pócimas & 10.77 \\
\hline Rezos & 4.62 \\
\hline Emplastos de hierbas & 4.62 \\
\hline Otros & 7.69 \\
\hline Ninguno & 43.08 \\
\hline
\end{tabular}

Fuente. Elaboración propia.

Atendiendo a las características económicas de la población estudiada, se observa que el sector agrícola es el más vulnerable ante el accidente ofídico. Durante estas labores agrarias se tiende a exponer los miembros superiores de los trabajadores, como consecuencia, en un $70.77 \%$ de los casos estudiados, es allí donde se presenta la mordedura del animal.

Además, se encontró una mala clasificación de la severidad del accidente en el paciente. De los 65 pacientes analizados, 53 fueron clasificados como leves, 11 pacientes como moderados, ningún caso se reportó como severo y en un caso no se reportó la severidad.
Cuando se realizó la reclasificación ajustada al protocolo dado por el Ministerio de Salud a nivel nacional, se encontró que un $20 \%$ de los pacientes fueron mal clasificados, 49 pacientes eran casos leves, 9 pacientes eran moderados y 6 pacientes se consideraban casos severos (Tabla 4).

Tabla 4. Clasificación de la severidad

\begin{tabular}{|l|c|c|}
\hline Clasificación & $\begin{array}{c}\text { Número de } \\
\text { pacientes según } \\
\text { historia clínica }\end{array}$ & $\begin{array}{c}\text { Número de } \\
\text { pacientes según } \\
\text { protocolo }\end{array}$ \\
\hline Leve & 53 & 49 \\
\hline Moderado & 11 & 9 \\
\hline Severo & 0 & 6 \\
\hline No reporta & 1 & 1 \\
\hline
\end{tabular}

Fuente. Elaboración propia.

Entre los pacientes que contaban con una buena clasificación según el protocolo dado por el Ministerio de Salud, de los 49 clasificados como leves, solo 28 recibieron el tratamiento adecuado; de los 9 clasificados como moderados, solo 2 recibieron un tratamiento adecuado; y de los 6 pacientes clasificados como severos, 5 recibieron un tratamiento adecuado (Tabla 5).

Tabla 5. Esquema de tratamiento recibido.

\begin{tabular}{|l|c|c|}
\hline Clasificación & $\begin{array}{c}\text { Número de } \\
\text { pacientes según } \\
\text { protocolo }\end{array}$ & $\begin{array}{c}\text { Número de } \\
\text { pacientes con tra- } \\
\text { tamiento adecuado }\end{array}$ \\
\hline Leve & 49 & 28 \\
\hline Moderado & 9 & 2 \\
\hline Severo & 6 & 5 \\
\hline
\end{tabular}

Fuente. Elaboración propia.

La familia de serpiente predominante en esta región es la perteneciente a los Bothrópicos: Mapaná y Patoco. El $26.15 \%$ de los pacientes no identifica el tipo de serpiente que lo atacó, lo que conlleva a una mala clasificación y ejecución del tratamiento. 
Los casos de accidente ofídico, analizados en San Roque - Antioquia durante los años 2008 y 2014 , permiten concluir que se presenta una mala clasificación, y, por consiguiente, un mal manejo de los casos; ya que el reporte generado por las entidades de salud de la región no diagnostica severidad en los pacientes atendidos y según la reclasificación de esta investigación, seis de estos pacientes fueron considerados severos por las manifestaciones clínicas.

\section{Discusión}

Teniendo en cuenta los resultados de estudios anteriores, concluimos que más del $30 \%$ de los accidentes ofídicos se presentan en edad reproductiva, con un pico máximo presentado a los 30 años de edad, afectan aún más al sexo masculino y están relacionados directamente con la presentación en el área rural, siendo esta de un $85 \%$. Vemos que, también, hay una relación entre la ocurrencia del accidente ofídico, con el tipo de actividad realizada en el momento del accidente, puesto que corresponde a las características económicas de la región. Las extremidades superiores son las más afectadas, con una frecuencia de un $70.77 \%$ de los casos, relacionándose de manera directa con la protección que utilizan los agricultores para su trabajo en miembros inferiores, al dejar expuestos los miembros superiores.

Se encontró que la información dada a la comunidad como promoción y prevención frente al accidente ofídico sigue siendo deficiente, pues según los últimos protocolos y las guías encontradas, la única práctica inicial aceptada es la inmovilización del miembro afectado; sin embargo, se encontró que lo más realizado por la población es la colocación de un torniquete en un $29.23 \%$.

Se encontró coherencia entre los reportes de la literatura frente a la familia de serpiente más implicada en accidente ofídico, pues el porcentaje de accidentes causados por la Bothrops corresponde a un $70.77 \%$.

Una de las grades problemáticas encontradas en el estudio es la mala clasificación clínica del paciente y su manejo, a pesar de que en el ámbito nacional contamos con protocolos de manejo del accidente ofídico. En resumen, un manejo adecuado sería dividir la atención del paciente en manejo prehospitalario y hospitalario. El primero se resume en medidas generales, como inmovilizar la extremidad en posición neutral, no realizar incisiones en el sitio de la mordedura, no utilizar torniquete, no succionar la herida, entre otros; pero lo principal es consultar rápidamente al hospital o centro de salud más cercano para iniciar el antídoto teniendo en cuenta el tipo de accidente (Campbell y Lamar, 2004; Otero et al., 1992; Otero, 1994; Otero, 2007).

Hablando del accidente Bothrópico y Lachésico, intrahospitalariamente se procederá a cumplir el protocolo: iniciar la infusión del antiveneno (monovalente o polivalente) sin previa prueba de sensibilidad. Todo paciente se considera un posible reactor y se inicia el goteo a una velocidad de 10-15 gotas por minuto, si en 10 minutos no hay reacciones, se finaliza la infusión en 30-60 minutos. La dosis varía según la clasificación del envenenamiento, así: leve: 2-4 ampollas; moderado: 4-8 ampollas; grave: 6-12 ampollas. En caso de mordedura por Bothrops $>1 \mathrm{~m}$ o verrugoso, aplicar 6 a 12 ampollas. Si tenemos un accidente tipo elapídico: todos los accidentes por corales se tratarán como graves, por lo que se administran 5 a 10 ampollas 
de antiveneno monovalente anticoral, y demás aplicación del protocolo. Si nos enfrentemos a un accidente crotálico son las mismas que en el accidente ofídico bothrópico y lachésico, con algunas consideraciones especialmente en la dosis del antiveneno, según sea el utilizado (Otero et al., 1992; Otero, 2007; Otero, 1994; Cuesta et al., 2010; Paredes, 2011).

\section{Agradecimientos}

Se hace mención y se resalta a la Uniremington como institución promotora de investigación, al hospital municipal de San Roque - Antioquia, como prestador de información y como fuente para la recolección de datos; finalmente, a los asesores de investigación y médicos por la buena disposición y haber permitido este aporte a la ciencia médica para mejorar en aspectos tratados.

\section{Referencias}

- Campbell, J., y Lamar, W. (2004). The Venomous Reptiles of the Western Hemisphere. Ithaka: Cornell University Press.

- Cuesta, J. et al. (2010). Animales ponzoñosos. Fundamentos de Medicina. Toxicología Clínica. $1^{\text {a }}$ Edicion. Medellín, Colombia: Corporación para Investigaciones Biológicas (CIB).

- Fry, B. G., y Wuster, W. (2004). Assembling an arsenal: origin and evolution of the snake venom proteome inferred from phylogenetic analysis of toxin sequences. Mol Biol Evols, 21(5), 870-83.

Lima, D. De, Álvarez Abreu, P., Freitas, C. De, Oliveira Santos, D., Oliveira Borges, R., Santos, T. Dos, Mendes Cabral, L., Rodrigues. C., y Castro, C. (2005). Snake Venom: Any Clue for Antibiotics and CAM? Evid Based Complement. Alternat Med, 2(1), 39-47.

- Otero, R., Tobón, G., Gómez, L., Osorio, R., Valderrama, R., Hoyos, D., Urreta, J., Molina, S., y Arboleda, J. (1992). Accidente ofídico en Antioquia y Chocó Aspectos clínicos y epidemiológicos, marzo de 1989 - febrero de 1990. Acta Médica Colombiana, 17(4), 229-249.

- Otero, R. (1994). Manual de Diagnóstico y Tratamiento del accidente ofídico. Medellín: Editorial Universidad de Antioquia.

- Otero, R. y Mesa, M. (2001). Mordeduras graves por serpientes. En: Quevedo A, Martínez y, Duque J, Mejía J, editor. El niño en estado crítico (pp. 571-78). Medellín: CIB.

- Otero, R., Gutiérrez, J., Mesa, B., Duque, E., Rodríguez, O., Arango, L., Gómez, F., Toro, A., Cano, F., Rodríguez, M., Caro, E., Martínez, J., Cornejo, W., Gómez, M., Uribe, F., Cárdenas, S., Núñez, V., y Díaz, A. (2002). Complications of Bothrops, Porthidium, and Bothriechis snakebites in Colombia. A clinical and epidemiological study of 39 cases attended in a university hospital. Toxicon, 40(8),1107-114.

- Otero-Patiño, R. (2007). Picaduras y mordeduras de animales ponzoñosos. En HUSVP (Ed.), Libro del VI seminario Urgente Saber de Urgencias, HUSVP y otros. Medellín: Ed. Artes y Letras Ltda. 
- Otero, R. (2007). Envenenamiento ofídico. En J. Correa, J. F. Gómez y R. Posada (Eds.), Fundamentos de Pediatría (3 ${ }^{\mathrm{a}}$ ed.). Medellín: CIB.
Paredes, Andrea. (2011). Informe del evento accidente ofídico, hasta el periodo epidemiológico 13 de 2011 - Boletín epidemiológico. Instituto Nacional de Salud de Colombia. 